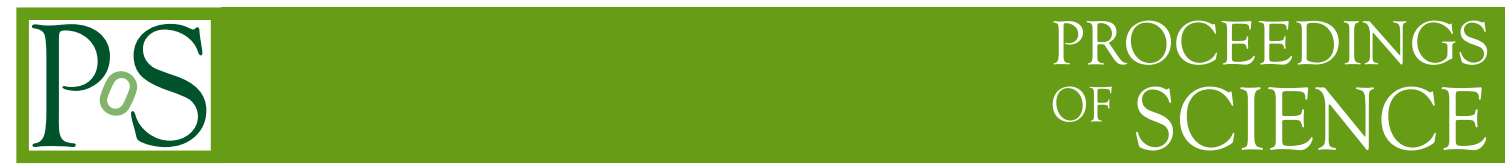

\title{
Nucleon Spin Structure in the Resonance Region
}

\section{Sarah K. Phillips*†}

University of New Hampshire

E-mail: Sarahp@jlab.org

The CLAS EG4 experiment was performed at Jefferson Lab to measure the generalized GDH sum for the proton and the neutron at low $Q^{2}$ from 0.015 to $0.5(\mathrm{GeV} / \mathrm{c})^{2}$. In addition to the spin structure functions that can be obtained from the inclusive measurement, the same data can be used to extract pion electroproduction asymmetries in the resonance region through the analysis of exclusive channels. Upon completion of the analysis, both sets of results will give a good test of chiral perturbation theory predictions in the resonance region. An overview of the EG4 experiment is given here, as well as preliminary results for the exclusive analysis.

6th International Workshop on Chiral Dynamics

July 6-10 2009

Bern, Switzerland

\footnotetext{
*Speaker.

$\dagger$ On behalf of the Jefferson Lab CLAS EG4 Collaboration.
} 


\section{Introduction}

For about 30 years, the spin structure of the nucleon has been investigated using lepton and photon beams. Spin structure function measurements at large $Q^{2}$ (deep inelastic scattering region) have provided information for the understanding of nucleon structure in terms of the elementary constituents of quantum chromodynamics (QCD), quarks and gluons [1, 2, 3]. However, much less is known in the low $Q^{2}$ region $\left(Q^{2}<1-2(\mathrm{GeV} / c)^{2}\right)$, where the perturbative techniques used at high energy fail and nucleon resonances start to play a dominant role. In this non-perturbative region, chiral perturbation theory $(\chi \mathrm{PT})$ is used for the calculation of moments of spin structure functions and other observables at very low momentum transfers $\left(Q^{2}<0.05-0.1(\mathrm{GeV} / c)^{2}\right)$.

Spin-dependent sum rules such as the Gerasimov-Drell-Hearn (GDH) sum rule [4, 5] are important tools to study nucleon spin structure. The GDH sum rule relates the helicity-dependent total absorption cross sections of circularly polarized photons on linearly polarized nucleons to the anomalous magnetic moment of the nucleon $\kappa$ :

$$
\int_{t h r}^{\infty}\left(\sigma_{1 / 2}-\sigma_{3 / 2}\right) \frac{d v}{v}=-2 \pi^{2} \alpha \frac{\kappa^{2}}{M^{2}}
$$

where $\sigma_{1 / 2}$ and $\sigma_{3 / 2}$ are the photo-absorption cross sections with the photon helicity aligned antiparallel or parallel to the nucleon spin, $M$ is the nucleon mass, $v$ is the photon energy, $t h r$ is the value of $v$ at the pion production threshold, and $\alpha$ is the electromagnetic coupling constant.

Although originally derived at the real photon point, the GDH integral can be extended to virtual photon absorption. Using the relationship between the forward spin-dependent Compton scattering amplitude $S_{1}\left(v, Q^{2}\right)$ and the nucleon spin structure function $g_{1}\left(x, Q^{2}\right)$, the extended GDH sum rule can be expressed as [6]

$$
I_{G D H}\left(Q^{2} \geq 0\right) \equiv \frac{16 \pi^{2} \alpha}{Q^{2}} \int_{0}^{x_{t h r}} g_{1}\left(x, Q^{2}\right) d x \equiv \frac{16 \pi^{2} \alpha}{Q^{2}} \Gamma_{1}=2 \pi^{2} \alpha S_{1}\left(0, Q^{2}\right)
$$

where $x=\frac{Q^{2}}{2 M v}$, and $\Gamma_{1}\left(Q^{2}\right)$ is the first moment of $g_{1}$, which in deep inelastic scattering is related to the fraction of the nucleon spin carried by the quarks. Note that at $Q^{2}=0$, the original GDH sum rule is recovered as $I_{G D H}\left(Q^{2}=0\right)=-\frac{1}{4} \kappa^{2}$, and as $Q^{2} \rightarrow \infty$, the Bjorken sum rule [7] becomes the constraint.

By extending the sum rule to finite $Q^{2}$, there is the advantage of a well-defined value of the integral at any momentum transfer, making this sum rule a good way to investigate nucleon spin structure. At low values of $Q^{2}, S_{1}\left(0, Q^{2}\right)$ can be calculated in chiral perturbation theory, giving predictions for the behavior of the GDH integral. A low $Q^{2}$ measurement of the GDH integral would be very useful to test the calculations done in the region where resonances have a significant contribution. The $Q^{2}$ dependence of $S_{1}\left(0, Q^{2}\right)$ can be also calculated using lattice QCD at intermediate $Q^{2}$ values, and with perturbative $\mathrm{QCD}$ at high $Q^{2}$, providing a way to study how nucleon structure evolves from the non-perturbative to the perturbative regime.

Measurements of the GDH sum have been done at different laboratories using photon beams (for the GDH sum rule) or electron beams (for the extended GDH sum rule). In electron scattering, the polarized inclusive electron-nucleon cross section can be written in terms of the spin structure 
functions $g_{1}\left(x, Q^{2}\right)$ and $g_{2}\left(x, Q^{2}\right)$ as

$$
\left(\frac{d \sigma^{\rightarrow \Leftarrow}}{d E^{\prime} d \Omega}\right)-\left(\frac{d \sigma^{\rightarrow \Rightarrow}}{d E^{\prime} d \Omega}\right)=\frac{4 \alpha^{2}}{Q^{2}} \frac{E^{\prime}}{M E v}\left[\left(E+E^{\prime} \cos \theta\right) g_{1}-2 M x g_{2}\right],
$$

where $E$ and $E^{\prime}$ are the incoming and outgoing electron energy. The measurement of this cross section gives direct access to the spin structure functions, particularly $g_{1}$.

Measurements of the generalized GDH sum for the proton and deuteron (neutron) were done by the EG4 experimental run group [8] in Hall B at Thomas Jefferson National Accelerator Facility. A similar measurement has been done using CLAS in Hall B by the EG1 experiment [9], at $Q^{2}=$ 0.05 to $5(\mathrm{GeV} / \mathrm{c})^{2}$, which added greatly to the world data available for $g_{1}$ for the proton and deuteron, covering both resonance and deep inelastic regions. The EG1 results for $g_{1}^{p}$ show that at low $Q^{2}$, the $\Delta(1232)$ resonance drives the asymmetry (and thus $g_{1}$ ) to negative values; as $Q^{2}$ increases, $g_{1}$ changes to the positive DIS limit for nearly all $x$ except for in the region of the $\Delta(1232)$ resonance [3]. The EG1 run group has also reported on the moment $\Gamma_{1}$ for the proton and the deuteron [10]. Other low $Q^{2}$ measurements have been done for the neutron at Jefferson Lab, using a polarized ${ }^{3} \mathrm{He}$ target in Hall A by the E94-010 experiment, for the generalized GDH sum rule [11] and the first moment [12], as well as by the E97-110 experiment [13].

Moments of the spin structure functions that involve higher powers of $x$ are also interesting, and their additional $x$-weighting emphasizes the kinematic region that is measured at Jefferson Lab. The forward spin polarizabilities of the nucleon can be extended to finite $Q^{2}$, leading to the concept of generalized polarizabilities [14]. The generalized forward spin polarizabilities $\gamma_{0}$ and $\delta_{L T}$ can be expressed in terms of the spin structure functions $g_{1}$ and $g_{2}$ [15]:

$$
\begin{aligned}
\gamma_{0}\left(Q^{2}\right) & =\frac{16 \alpha M^{2}}{Q^{6}} \int_{0}^{x_{0}} x^{2}\left[g_{1}\left(x, Q^{2}\right)-\frac{4 M^{2}}{Q^{2}} x^{2} g_{2}\left(x, Q^{2}\right)\right] d x, \\
\delta_{L T}\left(Q^{2}\right) & =\frac{16 \alpha M^{2}}{Q^{6}} \int_{0}^{x_{0}} x^{2}\left[g_{1}\left(x, Q^{2}\right)+g_{2}\left(x, Q^{2}\right)\right] d x .
\end{aligned}
$$

These quantities should be an ideal way to test calculations of chiral perturbation theory at low $Q^{2}$, especially as $\gamma_{0}$ is sensitive to resonances, but $\delta_{L T}$ is insensitive to the dominating $\Delta$ resonance. However, significant discrepancies exist between $\chi \mathrm{PT}$ calculations and the EG1 proton data for the generalized polarizability $\gamma_{0}^{p}$ [10], and although $\chi \mathrm{PT}$ calculations are not inconsistent with the E94-010 neutron data and $\gamma_{0}^{n}$, there is a significant discrepancy with the longitudinal-transverse polarizability $\delta_{L T}[16]$. Clearly, more measurements are needed to help solve this puzzle.

\section{The CLAS EG4 Experiment at Jefferson Lab}

The primary goal of the EG4 experiment is to extend our knowledge of the proton and neutron spin structure through measurements of the generalized GDH sum for the proton and the neutron (deuteron) at very low $Q^{2}\left(0.015-0.5(\mathrm{GeV} / \mathrm{c})^{2}\right)$. The experiment will extract $g_{1}$ from the measurement of the helicity-dependent inclusive cross sections. The results of this measurement will be used to test chiral perturbation theory predictions in the resonance region.

The EG4 experiment [8] took data in experimental Hall B at Jefferson Lab from February to May 2006. Polarized electrons were scattered off a polarized target down to $6^{\circ}$ scattering angles 

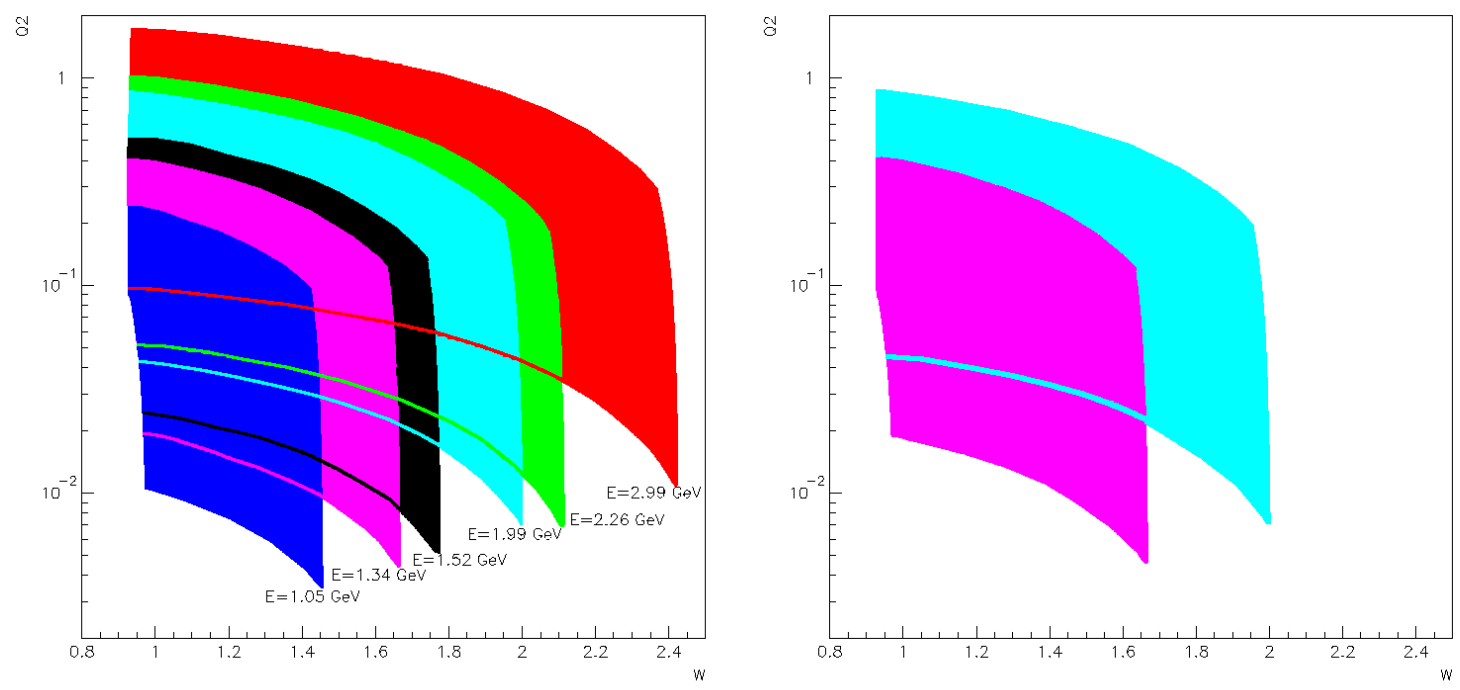

Figure 1: Kinematic coverage plots showing $Q^{2}$ (in $(\mathrm{GeV} / c)^{2}$ ) versus $W$ (in $\mathrm{GeV}$ ) for the polarized $\mathrm{NH}_{3}$ target (left plot) and the polarized $\mathrm{ND}_{3}$ target (right plot) in the EG4 experiment. From bottom to top, the beam energies used for the $\mathrm{NH}_{3}$ target are: 1.05, 1.34, 1.52, 1.99, 2.26, and $2.99 \mathrm{GeV}$; the beam energies for the $\mathrm{ND}_{3}$ target are 1.34 and $1.99 \mathrm{GeV}$.

and were detected in the CEBAF Large Acceptance Spectrometer (CLAS) [17]. The longitudinally polarized electron beam delivered by the CEBAF accelerator was produced by photoemission from a strained GaAs source and had a typical polarization of about $80 \%$ and various beam energies between $1-3 \mathrm{GeV}$. The experiment made use of two solid targets polarized using the dynamical nuclear polarization techinique: ${ }^{15} \mathrm{NH}_{3}$ for polarized protons, with a longitudinal polarization of about $70 \%-90 \%$, and ${ }^{15} \mathrm{ND}_{3}$ for polarized deuterons, with a longitudinal polarization of about $30 \%-45 \%$. The experiment also used a ${ }^{12} \mathrm{C}$ target and an empty cell target to perform background measurements.

The experiment used CLAS, the large solid angle spectrometer of Hall B, which consists of drift chambers, Čerenkov detectors, time-of-flight counters, and electromagnetic calorimeters, as well as a new Čerenkov counter built specifically for the measurement. The azimuthal acceptance of CLAS is divided into six sectors, each covering $\pi / 3$ for the scattered particles. The new Čerenkov detector was built and commissioned by the INFN-Genova group, and was necessary to achieve the uniform detection efficiency at forward angles required to do an absolute cross section measurement at low $Q^{2}$. The detector was designed for a high electron efficiency (of the order of $99.9 \%$ ) and a high pion rejection ratio (of the order of $10^{-3}$ ), and was installed in sector 6 of CLAS. The signals from the new Čerenkov detector were built into the main electron trigger; only this sixth of the azimuthal acceptance of CLAS was used to detect scattered electrons.

Plots of the kinematic coverage for both of the polarized targets are shown in Figure 1. The data were taken at beam energies of $1.05,1.34,1.52,1.99,2.26$, and $2.99 \mathrm{GeV}$ with the $\mathrm{NH}_{3}$ target, covering a kinematic range of $0.015<Q^{2}<0.5(\mathrm{GeV} / \mathrm{c})^{2}$, and 1.34 and $1.99 \mathrm{GeV}$ for the $\mathrm{ND}_{3}$ target, which covers a kinematic range of $0.015<Q^{2}<0.2(\mathrm{GeV} / c)^{2}$.

The analysis on the inclusive $\left(e, e^{\prime}\right)$ channel is still underway, with preliminary results expected in the late fall. Figure 2 shows the anticipated results for the proton $\Gamma_{1}$ (left plot) and for the neutron 

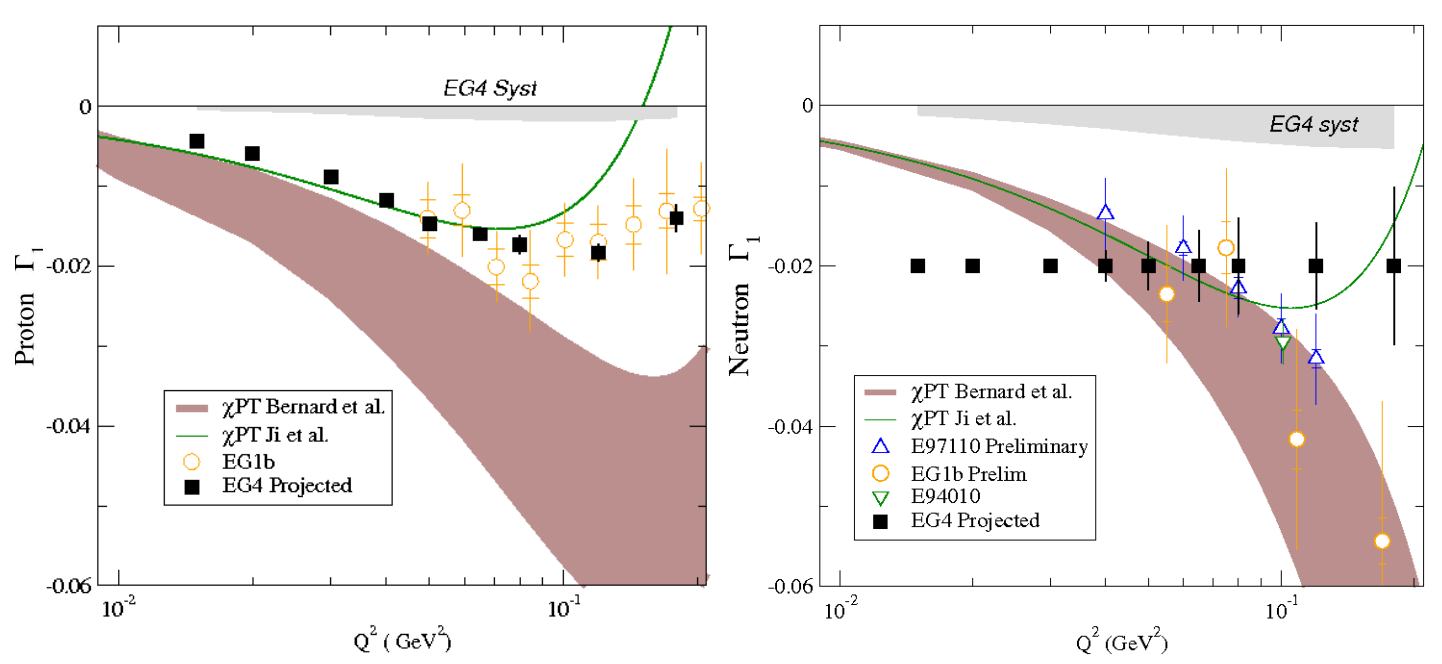

Figure 2: The projected results for $\Gamma_{1}^{p}$ (left plot) and $\Gamma_{1}^{n}$ (right plot) from the EG4 experiment, shown with previous results from CLAS EG1b [10, 18], Hall A E94-010 [11, 12, 16], and Hall A E97-110 [13], as well as the $\chi$ PT calculations by Bernard et al. [19] and Ji et al. [20].

$\Gamma_{1}$ (right plot). These projected results are shown in comparison with the proton results from the CLAS EG1b experiment [10], the neutron results from Hall A E94-010 [11, 12, 16], the preliminary neutron results from CLAS EG1b [18] and Hall A E97-110 [13], and the $\chi$ PT calculations by Bernard et al. [19] and Ji et al. [20].

\subsection{Exclusive Channel Analysis}

The data include multiparticle final states, making it possible to study exclusive channels as well. The dominant physics channel of interest is the extraction of the pion electroproduction asymmetries in the nucleon resonance region. The extracted asymmetries can also be compared to chiral perturbation theory predictions at low $Q^{2}$ and can help to constrain phenomenological models such as MAID [21] and SAID [22].

A diagram of the kinematics for such exclusive channels is shown in Figure 3. For a polarized beam, $P_{e}$, and a polarized target, $P_{t}$, the pion electroproduction cross section can be written as

$$
\frac{d \sigma}{d \Omega_{\pi}^{*}}=\frac{|\vec{q}|}{q_{\gamma}^{C M}}\left\{\frac{d \sigma_{0}}{d \Omega_{\pi}^{*}}+P_{e} \frac{d \sigma_{e}}{d \Omega_{\pi}^{*}}+P_{t} \frac{d \sigma_{t}}{d \Omega_{\pi}^{*}}-P_{e} P_{t} \frac{d \sigma_{e t}}{d \Omega_{\pi}^{*}}\right\}
$$

where $\sigma_{0}$ is the unpolarized cross section, $\sigma_{e(t)}$ is the cross section for a polarized beam (target), and $\sigma_{e t}$ is the cross section for both the beam and target polarized [24, 25]. Three spin asymmetries can be defined, a single-beam asymmetry, $A_{e}$, a single-target asymmetry, $A_{t}$, and a double beam-target asymmetry, $A_{e t}$ :

$$
A_{e}=\frac{\sigma_{e}}{\sigma_{0}}, \quad A_{t}=\frac{\sigma_{t}}{\sigma_{0}}, \quad A_{e t}=\frac{\sigma_{e t}}{\sigma_{0}}
$$

The single-beam asymmetry $A_{e}$ can be accessed from measurements with unpolarized targets; the goal of this exclusive channel analysis for EG4 is the extraction of the single-target asymmetry $A_{t}$ and the double spin beam-target asymmetry $A_{e t}$ for the charged and neutral pion channels. 
Preliminary asymmetries for $A_{t}$ and $A_{e t}$ have been extracted from the $3 \mathrm{GeV} \mathrm{NH}_{3}$ data for the $\left(e, e^{\prime} \pi^{+}\right) n$ channel, which are shown in Figure $\bigoplus$. The figure shows these preliminary results for $A_{t}$ and $A_{e t}$ as functions of $\phi^{*}$, integrated over a range of $1.34<W<1.58 \mathrm{GeV}$. These asymmetries have not yet been corrected for variations in the CLAS acceptance, nor for the contribution from unpolarized nucleons in the target. However, the data indicate that about $20 \%$ of the events are from polarized protons in the $\mathrm{NH}_{3}$ target, so the predictions from the phenomenological models MAID2007 and DMT [26, 27] have been scaled by 0.20 for a comparison to these preliminary results.

The exclusive channel analysis is well underway, with the corrections for the non-uniform acceptance of CLAS nearly completed. These results of this analysis will extend the $Q^{2}$ range of our knowledge of resonant pion electroproduction asymmetries down to $Q^{2} \approx 0.015(\mathrm{GeV} / c)^{2}$ and allow for a comparison to $\chi \mathrm{PT}$ predictions.

\section{Future Measurement: E08-027}

In addition to the EG4 experiment, E08-027 has been approved to run in JLab Hall A in 2011 [28]. This experiment will perform an inclusive measurement to determine the $g_{2}^{p}$ structure function in the resonance region for $0.02<Q^{2}<0.4(\mathrm{GeV} / c)^{2}$. These data will be used to evaluate the Burkhardt-Cottingham sum rule [29] and the longitudinal-transverse polarizability $\delta_{L T}$. The kinematic region of this measurement will complement the EG4 measurement of $g_{1}^{p}$ and $g_{1}^{d}$.

\section{Summary}

Our understanding of nucleon structure is not complete, if we do not have a thorough knowledge of the nucleon's spin response in the resonance region in the domain of $\chi$ PT. The JLab CLAS EG4 experiment will report results for the generalized GDH sum rule at low $Q^{2}$ from its inclusive measurement, and pion electroproduction asymmetries in the resonance region from its exclusive analysis. These results will give insight into the behavior of nucleon structure from the

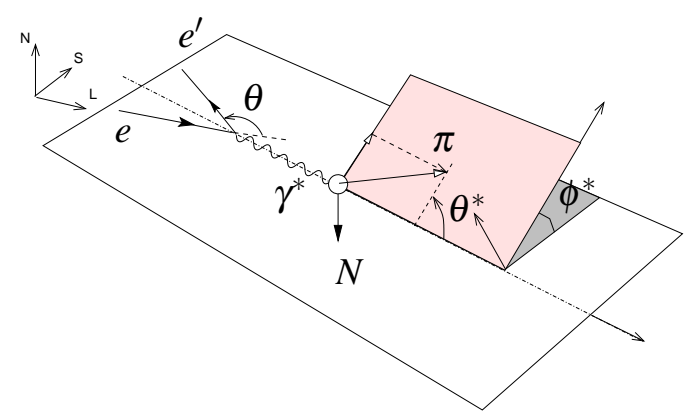

Figure 3: Diagram of pion electroproduction kinematics. The incident and scattered electrons define the scattering plane, and the hadronic reaction plane is given in the $\gamma^{*} N$ center-of-mass system by the pion and nucleon momenta. The kinematics is defined by the four-momentum transfer $Q^{2}$, the invariant mass of the $\gamma^{*} N$ system $W$, and the azimuthal and the polar angles of the pion in the $\gamma^{*} N$ center-of-mass frame $\theta^{*}$ and $\phi^{*}[23]$. 
(a) $A_{t}$
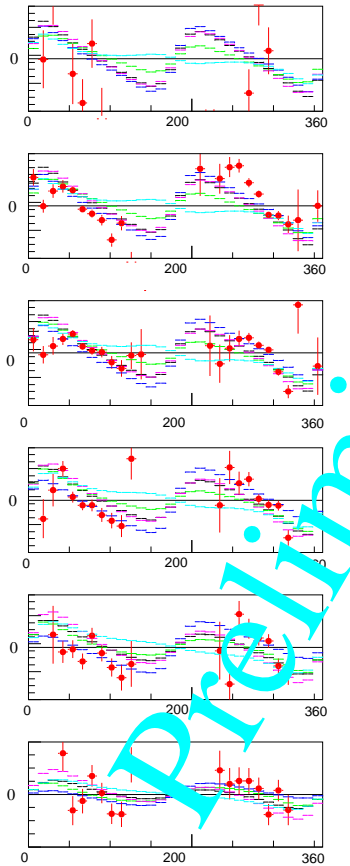
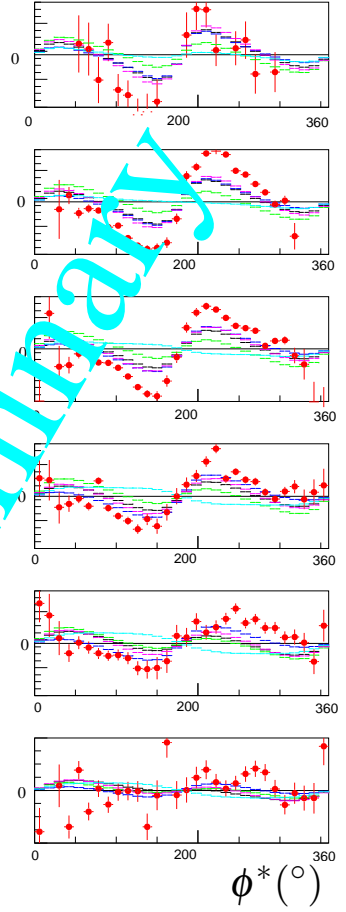

(b) $A_{e t}$
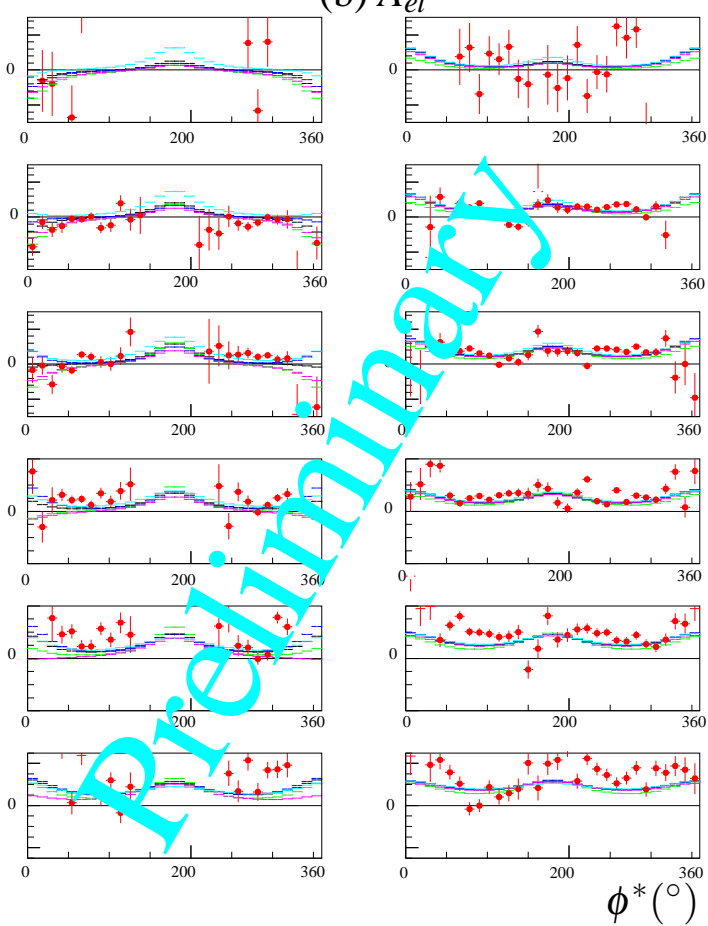

Figure 4: Preliminary asymmetries for $A_{t}$ (left panel) and $A_{\text {et }}$ (right panel) from the $3 \mathrm{GeV}$, polarized $\mathrm{NH}_{3}$ target data are shown in red as a function of the pion $\phi^{*}$ for $1.34<W<1.58 \mathrm{GeV}$ along with predictions from DMT (green), MAID2007 full calculation (black), and MAID2007 with $P_{11}$ (blue), $S_{11}$ (magenta), and $D_{13}$ (cyan) turned off. The asymmetries on the left have been integrated over $\cos \theta^{*}=(0,0.5)$ and on the right over $\cos \theta^{*}=(0.5,1.0)$, for each panel respectively. The six rows from top to bottom correspond to different integration ranges in $Q^{2}$ : $(0.054,0.0919),(0.0919,0.156),(0.156,0.266),(0.266,0.452),(0.452$, $0.770)$, and $(0.77,1.31)(\mathrm{GeV} / \mathrm{c})^{2}$. The asymmetries have not been corrected for spectrometer acceptance variations, and all predictions have been scaled by 0.20 to approximately correct for contributions from unpolarized nucleons in the target [23].

non-perturbative to the perturbative region, and give a good test of $\chi \mathrm{PT}$ predictions in the resonance region.

\section{References}

[1] B. W. Filippone and X. D. Ji, Adv. Nucl. Phys. 26, 1 (2001) [arXiv:hep-ph/0101224].

[2] J. P. Chen, A. Deur and Z. E. Meziani, Mod. Phys. Lett. A 20, 2745 (2005) [arXiv:nucl-ex/0509007].

[3] S. E. Kuhn, J. P. Chen and E. Leader, Prog. Part. Nucl. Phys. 63, 1 (2009) [arXiv:0812.3535 [hep-ph]].

[4] S. B. Gerasimov, Sov. J. Nucl. Phys. 2, 430 (1966) [Yad. Fiz. 2, 598 (1966)].

[5] S. D. Drell and A. C. Hearn, Phys. Rev. Lett. 16, 908 (1966).

[6] X. D. Ji and J. Osborne, J. Phys. G 27, 127 (2001) [arXiv:hep-ph/9905410].

[7] J. D. Bjorken, Phys. Rev. 148, 1467 (1966). 
[8] JLab E03-006, M. Battaglieri, R. De Vita, A. Deur and M. Ripani spokespersons; JLab PR05-111, A. Deur, G. Dodge and K. Slifer spokespersons.

[9] K. V. Dharmawardane et al. [CLAS Collaboration], Phys. Lett. B 641, 11 (2006) [arXiv:nucl-ex/0605028].

[10] Y. Prok et al. [CLAS Collaboration], Phys. Lett. B 672, 12 (2009) [arXiv:0802.2232 [nucl-ex]].

[11] M. Amarian et al., Phys. Rev. Lett. 89, 242301 (2002) [arXiv:nucl-ex/0205020].

[12] M. Amarian et al. [Jefferson Lab E94-010 Collaboration], Phys. Rev. Lett. 92, 022301 (2004) [arXiv:hep-ex/0310003].

[13] V. A. Sulkosky, AIP Conf. Proc. 1155, 93 (2009).

[14] P. A. M. Guichon, G. Q. Liu and A. W. Thomas, Nucl. Phys. A 591, 606 (1995) [arXiv:nucl-th/9605031].

[15] D. Drechsel, B. Pasquini and M. Vanderhaeghen, Phys. Rept. 378, 99 (2003) [arXiv:hep-ph/0212124].

[16] M. Amarian et al. [Jefferson Lab E94010 Collaboration], Phys. Rev. Lett. 93, 152301 (2004) [arXiv:nucl-ex/0406005].

[17] B. A. Mecking et al. [CLAS Collaboration], Nucl. Instrum. Meth. A 503 (2003) 513.

[18] http://www.jlab.org/ kuhn/EG2000intro.html.

[19] V. Bernard, T. R. Hemmert and U. G. Meissner, Phys. Rev. D 67, 076008 (2003) [arXiv:hep-ph/0212033].

[20] X. D. Ji, C. W. Kao and J. Osborne, Phys. Lett. B 472, 1 (2000) [arXiv:hep-ph/9910256].

[21] D. Drechsel, O. Hanstein, S. S. Kamalov and L. Tiator, Nucl. Phys. A 645, 145 (1999) [arXiv:nucl-th/9807001]; http://wwwkph.kph.uni-mainz.de/MAID/.

[22] The SAID Partial-Wave Analysis, R. A. Arndt et al., http://gwdac.phys.gwu.edu/

[23] X. Zheng, AIP Conf. Proc. 1155, 135 (2009).

[24] A. S. Raskin and T. W. Donnelly, Annals Phys. 191, 78 (1989) [Erratum-ibid. 197, 202 (1990)].

[25] A. Bartl and W. Majerotto, Nucl. Phys. B 62, 267 (1973).

[26] S. S. Kamalov, S. N. Yang, D. Drechsel, O. Hanstein and L. Tiator, Phys. Rev. C 64, 032201 (2001) [arXiv:nucl-th/0006068].

[27] S. S. Kamalov and S. N. Yang, Phys. Rev. Lett. 83, 4494 (1999) [arXiv:nucl-th/9904072].

[28] JLab E08-027, A. Camsonne, J. P. Chen, and K. Slifer, spokespersons; http://hallaweb.jlab.org/experiment/E08-027/.

[29] H. Burkhardt and W. N. Cottingham, Annals Phys. 56, 453 (1970). 